\title{
Attitude à adopter face à des patients incapables de discernement
}

Les directives médico-éthiques publiées par I'ASSM ces dernières années contiennent des dispositions différentes concernant les droits des patients. C'est pourquoi, en mai 2004, la Commission centrale d'éthique (CCE) a chargé un groupe de travail d'élaborer un corps de texte homogène pour les futures directives médico-éthiques. Le groupe de travail «Droits des patients» s'est fondé en premier lieu sur les dispositions du droit fédéral et la liberté de décision qu'il permet; par ailleurs, il a tenu compte de la Convention bioéthique européenne ainsi que des principes généraux de l'éthique médicale. En automne 2005, le groupe de travail a présenté son projet de «Principes de base médico-éthiques pour le droit des patientes et patients à l'autodétermination»; ceux-ci ont été approuvés par le Sénat le 24 novembre 2005. L'essentiel des contenus de ces principes de base est résumé ci-après.

Prof. Dr iur. Dr h.c. Kurt Seelmann ${ }^{a}$, responsable du groupe de travail «Droits des patients»

a Professeur ordinaire de droit pénal et philosophie du droit, Université de Bâle

Correspondance:

lic. iur. Michelle Salathé Académie Suisse des Sciences Médicales Petersplatz 13 CH-4051 Bâle

m.salathe@samw.ch

\section{Signification des consentements de substitution}

Le traitement et la prise en charge de patients capables de discernement ne sont légitimes que s'il existe un consentement valable. Pour les actes médicaux sur des personnes incapables de donner elles-mêmes leur consentement, on doit, par la force des choses, se baser sur des consentements de substitution, qui peuvent être: une décision de l'autorité tutélaire, un consentement écrit rédigé à une date antérieure («directives anticipées»), un consentement donné par des représentants légaux ou par un représentant désigné par le patient, ou un consentement présumé.

S'il s'agit de personnes qui n'ont jamais été capables de discernement (incapacité de discernement originaire), sont donc exclus d'emblée tous les consentements de substitution rattachés à une capacité de discernement antérieure, c'est-àdire les consentements donnés par le représentant désigné par le patient, ainsi que les directives anticipées. Dans ces circonstances, seuls restent possibles le recours à la représentation légale et les (autres) instruments du droit tutélaire. Le représentant légal doit dans tous les cas avoir comme préoccupation principale le bien objectif - c'est-à-dire défini d'un point de vue médical - de la personne qu'il représente. Dans tous les autres cas, c'est-à-dire lorsqu'une capacité de discernement a précédé la phase d'incapacité (incapacité de discernement acquise), on peut en principe se baser sur tous les consentements de substitution cités plus haut. Mais se pose alors nécessairement la question de leur hiérarchisation dans les situations conflictuelles. A cet égard, le principe de subsidiarité valable pour le droit tutélaire devrait avoir pour conséquence qu'une décision de l'autorité tutélaire n'entre en ligne de compte que lorsque d'autres consentements de substitution ne remplissent pas leur tâche ou qu'il existe un conflit d'intérêts ou d'opinions. De plus, la pratique montre que vouloir faire intervenir en premier lieu l'autorité tutélaire pour des patients incapables de discernement n'est pas réalisable, car des décisions importantes sur le plan médical doivent souvent être prises dans des situations où le temps presse. Il arrive aussi souvent que les ressources en personnel des autorités tutélaires ne permettent pas à celles-ci d'assumer pleinement une fonction de représentation. Dans ces conditions, le personnel médical traitant reçoit l'instruction informelle d'agir en fonction de la volonté présumée du patient.

\section{Directives anticipées}

L'importance qu'ils convient d'accorder aux directives anticipées est particulièrement sujette à controverse. Pour les uns, ces directives doivent 
être absolument respectées, car elles expriment la volonté du patient. Pour les autres, elles constituent (seulement) un indice important dont on doit tenir compte lorsque l'on recherche la volonté présumée du patient.

Le groupe de travail est d'avis que les directives anticipées constituent un indice important dont on doit tenir compte lorsque l'on recherche la volonté présumée du patient: les directives anticipées constituent des dispositions préalables en vue d'une situation existentielle difficilement prévisible. On ne peut donc pas les comparer, ni d'un point de vue juridique, ni d'un point de vue éthique, à une déclaration faite le moment venu par le patient capable de discernement. Les exigences posées aux consentements exprimés antérieurement ne doivent donc pas être moins strictes que celles posées aux consentements donnés dans la situation même. A priori, on ne peut parler de consentement valable que lorsque le patient a été suffisamment informé. Or, les directives anticipées ne répondent pas toujours à ce critère: souvent, le patient rédige des directives anticipées sans avoir reçu la moindre information; et même s'il a reçu des informations, celles-ci, le temps passant, ne correspondent plus forcément à la situation présente; et même si elles y correspondent encore, il était impossible, au moment où elles ont été rédigées, d'anticiper le tableau clinique exact avec les bénéfices et les risques concrets de tel ou tel acte médical. En d'autres termes, lorsqu'il rédige des directives anticipées, le patient ne peut pas se projeter dans une situation future et prévoir les différentes options qui lui seront alors proposées.

\section{Représentant désigné par le patient}

Dans sa portée, la décision prise par un représentant désigné par le patient s'apparente à des directives anticipées. Elle constitue un indice (fort) dont on doit tenir compte lorsque l'on recherche la volonté présumée du patient le moment venu, mais elle ne peut pas non plus revêtir un caractère obligatoire exclusif, cela pour les raisons mentionnées à propos des directives anticipées. Elle se distingue néanmoins de la représentation légale en ce qu'elle résulte de l'autodétermination du patient. Voilà pourquoi, en cas d'incapa- cité de discernement acquise du patient et de désaccord entre le représentant désigné par le patient (alors qu'il était encore capable de discernement) et un représentant légal (désigné ultérieurement), priorité devrait être donnée en principe à la personne désignée par le patient (idée fondatrice du principe de subsidiarité). Lorsque son consentement est requis pour des actes médicaux, le représentant désigné par le patient doit, tout comme le représentant légal, se préoccuper avant tout du bien du patient. Il ne peut donc consentir qu'à des actes médicaux indiqués. Il peut toutefois, si le patient le lui a demandé, refuser des actes médicaux indiqués et indispensables d'un point de vue médical (dans une telle situation conflictuelle, le personnel médical peut s'adresser à l'autorité tutélaire).

\section{Consentement présumé}

Pour toutes ces raisons, le consentement présumé du patient revêt une importance capitale, en particulier lorsque l'on considère les directives anticipées comme un indice important dans le cadre d'un consentement présumé, eu égard à l'obligation, pour le représentant désigné, d'avoir en tous les cas comme préoccupation principale le bien du patient au moment de consentir à des actes médicaux, et compte tenu du fait que les instruments du droit tutélaire n'ont qu'une fonction subsidiaire.

En ce qui concerne le consentement présumé, on doit dans ces conditions décider qui est habilité en premier lieu à constater la volonté présumée du patient. Etant donné que cette constatation exige toujours de bien connaître à la fois les souhaits très personnels du patient et les composantes objectives du «bien» du patient, le groupe de travail propose de privilégier ici une décision prise par consensus entre les proches et le personnel médical traitant.

Ce faisant, on ne restreint pas le principe d'autodétermination; au contraire, on rend ce principe - à peu près - applicable à des patients incapables de discernement. Le contrôle mutuel de deux groupes chargés de définir la volonté présumée du patient est le meilleur moyen de garantir le respect de cette volonté. 